\title{
Periodontitis una enfermedad multifactorial: Diabetes Mellitus
}

Periodontitis a multifactorial disease: Diabetes Mellitus

Periodontite uma doença multifatorial: Diabetes Mellitus

DOI: http://dx.doi.org/10.23913/rics.v6i11.51

Fabian Enrique Aguilar Soto

Facultad de Odontología Campus Mexicali, Universidad Autónoma de Baja California, México kiqueaguilar1@gmail.com

Fernando Josafaht Sosa Morales Facultad de Odontología Campus Mexicali, Universidad Autónoma de Baja California, México josafaht_sosa@hotmail.com

Yolanda Bojórquez Anaya

Facultad de Odontología Campus Mexicali, Universidad Autónoma de Baja California, México yolandabojorquez@gmail.com

Zureya Fontes García Facultad de Odontología Campus Mexicali, Universidad Autónoma de Baja California, México zureyafontesgarcia@hotmail.com

\section{Resumen}

La periodontitis es una enfermedad multifactorial, en la cual la placa bacteriana es el factor iniciador, influyendo a la vez factores locales como sistémicos. La diabetes es una enfermedad sistémica debida a una disminución de la secreción de la hormona insulina o a una deficiencia de su acción.

Objetivo: el propósito de este trabajo es explicar algunos conceptos básicos que ayuden a la compresión de la asociación bidireccional de la enfermedad periodontal con Diabetes Mellitus, 
con base en los hallazgos científicos encontrados. Con el objetivo de conocer, tratar y cuidar la salud de un paciente diabético.

Metodología: se realizo una búsqueda bibliográfica en base de datos (pubmed, periodontology 2000, medline, scielo, elsevier, intramed, j periodontol, etc.) nacionales e internacionales, utilizando palabras claves.

Resultados: se escogieron 55 artículos para el estudio, destacando el objetivo de este trabajo que es la relación entre Diabetes Mellitus y enfermedad periodontal y viceversa.

Discusión/Conclusión: la asociación entre Diabetes Mellitus y periodontitis se ha reportado en numerosos estudios, al igual que en estudios en animales sugieren que la presencia de una condición tiende aumentar el riesgo y la severidad de la otra.

Palabras clave: Diabetes Mellitus, periodontitis, hiperlipidemia.

\section{Abstract}

Periodontitis is a multifactorial disease, in which plaque is the initiator factor, influencing both local and systemic factors. Diabetes is a systemic disease due to a decrease in the secretion of the hormone insulin or a deficiency of its action.

Objective: The purpose of this paper is to explain some basic concepts that help to understand the bi-directional association of periodontal disease with Diabetes Mellitus, based on the scientific findings. In order to know, treat and care for the health of a diabetic patient.

Methodology: a bibliographic search was carried out in national and international databases (pubmed, periodontology 2000, medline, scielo, elsevier, intramed, j periodontol, etc.) using key words.

Results: 55 articles were chosen for the study, highlighting the objective of this work is the relationship between Diabetes Mellitus and periodontal disease. 
Conclusion: The association between Diabetes Mellitus and periodontitis has been reported in numerous studies, as in animal studies suggest that the presence of one condition tends to increase the risk and severity of the other.

Key words: Diabetes Mellitus, periodontitis, hyperlipidemia.

\section{Resumo}

A periodontite é uma doença multifatorial em que a placa é o fator inicial que influencia fatores locais e sistêmicos. A diabetes é uma doença sistémica devido a uma diminuição da secreção da insulina ou da hormona a uma deficiência de acção.

Objetivo: O objetivo deste artigo é explicar alguns conceitos básicos que o ajudarão a compreensão da associação bidirecional da doença periodontal com Diabetes Mellitus, com base em descobertas científicas. A fim de compreender, tratar e cuidar da saúde de um paciente diabético.

Métodos: Um banco de dados de pesquisa literatura (PubMed, periodontia 2000, o Medline, Scielo, Elsevier, Intramed, j Periodontol, etc.) nacional e internacional, foi realizada utilizando palavras-chave.

Resultados: 55 artigos foram seleccionados para o estudo, com destaque para o objectivo deste trabalho é a relação entre a diabetes mellitus e doença periodontal e vice-versa.

Discussão / Conclusão: A associação entre diabetes mellitus e periodontite foi avaliado em diversos estudos, como os estudos em animais sugerem que a presença de uma condição, tende a aumentar o risco e gravidade da outra.

Palavras-chave: Diabetes Mellitus, periodontite, hiperlipidemia.

Fecha recepción: Junio $2016 \quad$ Fecha aceptación: Noviembre 2016 


\section{Introducción}

La periodontitis es una enfermedad multifactorial, la cual se caracteriza por una inflamación crónica causada por microorganismos bacterianos en una biopelícula, ocasionando destrucción progresiva del aparato de soporte dentario: perdida del ligamento periodontal, destrucción ósea, formación de bolsa periodontales, recesiones gingivales y pérdida dentaria.

La gingivitis y la periodontitis son enfermedades que son iniciadas por un desafío bacteriano con la inflamación reversible de la gingivitis progresando a la periodontitis destructiva, impulsada por una compleja respuesta desequilibrada del huésped al desafío bacteriano. Esta respuesta puede estar influenciada por una serie de factores de riesgo que afectarán la susceptibilidad al desarrollo de la enfermedad, la tasa de progresión de la enfermedad e incluso la respuesta al tratamiento.

(Miguel, P., Niño, A., Batista, K. 2016) La periodontitis es, sin duda, la mas significativa de estas enfermedades, pues causa perdida dental.

Se cree que las enfermedades periodontales son las condiciones inflamatorias crónicas más comunes en todo el mundo con una prevalencia global de periodontitis en los EE. UU. , estimada en el rango de $47 \%$ en adultos de 30 años o más. La Hoja Informativa sobre la Salud Oral de la Organización Mundial de la Salud (OMS) informa de una enfermedad periodontal severa, que puede resultar en pérdida de dientes, en el 15-20\% de los adultos de mediana edad (35-44).

Hoy en día y tras numerosos estudios epidemiológicos, se acepta el concepto de la existencia de determinados factores de riesgo que van a modular la susceptibilidad o resistencia del hospedador a padecer enfermedad periodontal, por lo tanto, en su desarrollo van a intervenir varias causas considerándose dicha patología de etiología multifactorial.

(Ghiotto G. et al. 2012) al igual que (Xiaodong, Z, Wenyi, Z., Xiaoli, L., Wu, Z., Yiming, L., 2015) mensionan que la Diabetes Mellitus (DM) es sin duda un grupo de trastornos metabólicos caracterizados por hiperglucemia causada por defectos en la secreción de insulina, acción de la insulina, o ambos. Existen dos tipos principales de diabetes: la diabetes tipo 1 y la forma más común, la diabetes tipo 2. 
Los datos en estudios epidemiológicos, así como todos los estudios en animales sugieren que la presencia de una condición tiende a aumentar el riesgo y la severidad de la otra. Es ampliamente aceptado que la Diabetes Mellitus es un factor de riesgo para el aumento de la prevalencia y la gravedad de periodontitis. (Tan, W., Tay, F., Lim, L, 2006).

Diabetes Mellitus es un factor de riesgo para periodontitis, a su vez, el control metabólico de los diabéticos se dificulta por la presencia de la infección periodontal, aumentando el riesgo de complicaciones crónicas y eventualmente, la mortalidad por diabetes (Jiménez, T., Licea, M.,2013).

\section{Enfermedad periodontal: multifactorial}

La periodontitis es una enfermedad multifactorial, causada por la presencia prolongada de una biopelícula subgingival, creando una inflamación crónica en los tejidos periodontales ocasionando destrucción de ellos. En la progresión de la enfermedad periodontal influyen una variedad de causas y factores de riesgo como: factores sociales y de comportamiento, psicológicos, factores genéticos, composición microbiana de la placa, factores dentales, factores endocrinos, enfermedades sistémicas, enfermedades genéticas, problemas de higiene, y sociodemográficos, aunque solo algunos de ellos tienen evidencia científica que muestran una relación causal con la periodontitis.

\section{Periodontitis y la relación con Diabetes Mellitus}

La asociación entre la Diabetes Mellitus y la periodontitis se ha reportado en numerosos estudios. En general se acepta que la interrelación entre la Diabetes Mellitus y la periodontitis es una relación de dos vías. Los datos de estudios de epidemiología, así como todos los estudios en animales sugieren que la presencia de una condición tiende a aumentar el riesgo y la severidad de la otra. Es ampliamente aceptado que la Diabetes Mellitus es un factor de riesgo para el aumento de la prevalencia y la gravedad de la periodontitis. A la inversa, periodontitis es un factor importante para el aumento de riesgo de complicaciones de la diabetes en pacientes con diabetes. (Jimenez, 2013; Herrera, SEPA; Dominguez 1993). 
Si bien la asociación epidemiológica entre la periodontitis y la diabetes esta siendo relativamente clara, los mecanismos biológicos de la asociación son todavía poco concluyentes. Los sujetos que presentaban DM, sobre todo con un estado glucémico mal controlada, tienen una periodontitis más severa y generalizada. Los esfuerzos por determinar los mecanismos que dan una mayor incidencia de periodontitis en la DM se centraron en las diferencias en la microbiota subgingival. Un informe sugiere que la hiperglucemia se asocia con una microbiota subgingival alterada (Piyamas, A. et al. 2013).

(Xiaodong, Z, et al. 2015) Mensiona una serie de estudios. Los estudios epidemiológicos sugieren generalmente un mayor riesgo de periodontitis entre las personas con Diabetes Mellitus. Nelson et al. (1990) encontraron en su estudio que, los indios Pima de Arizona tienen una muy alta prevalencia de la Diabetes Mellitus tipo 2. En un subgrupo de esta población de 15 a 54 años de edad, la prevalencia por edad y sexo ajustadas de la enfermedad periodontal fue de $60 \%$ en los pacientes diabéticos, mientras que la tasa fue del $36 \%$ en las de individuos no diabéticos. Del mismo modo, en un estudio entre los afroamericanos Gullah en Carolina del Sur, el 70,6\% de los pacientes diabéticos tenían periodontitis moderada, la cual era mucho más alta que la prevalencia de 10.6\% entre los afroamericanos sin diabetes (Fernandes et al. 2009). A nivel internacional, un estudio (Weinspach et al. 2013) realizado en la población de Alemania informó que los pacientes diabéticos tipo 2 tenían significativamente mayor índice de detección periodontal que los de los controles no diabéticos $(3.52$ vs $3.26, p<0,01) 0.8$. Un estudio (Al-Khabbaz et al. 2013) de casos y controles reveló que la enfermedad periodontal fue más evidente en los niños (4-14 años) con Diabetes Mellitus tipo 1 en Kuwait que los que no presentaban diabetes. El índice de placa, índice gingival y sangrado al sondaje fueron significativamente mayores en los pacientes con diabetes en comparación con los no diabéticos de control.

\section{Diabetes Mellitus}

La Organización Mundial de la Salud OMS (1999) dice que la diabetes sacarina es un trastorno metabólico que tiene causas diversas; se caracteriza por hiperglucemia crónica y trastornos del metabolismo de los carbohidratos, las grasas y las proteínas como consecuencia de anomalías de la secreción o del efecto de la insulina. Con el tiempo, la enfermedad puede causar daños, disfunción e insuficiencia de diversos órganos. 
Las dos formas principales de Diabetes Mellitus, tipo 1 y tipo 2, comparten manifestaciones clínicas, pero se caracterizan por tener una causalidad distinta. La diabetes tipo 1 se produce cuando las células BETA del páncreas se destruyen y se produce una cantidad insuficiente de insulina. En la mayor parte de los casos, la diabetes tipo 1 es el resultado de una inflamación de origen autoinmunitario, con destrucción y apoptosis de las células. Aproximadamente el $90 \%$ de los pacientes con diabetes presenta el tipo 2. El desarrollo de la diabetes tipo 2 se relaciona directamente con el aumento en la cantidad de tejido adiposo visceral. El tejido adiposo se considera un órgano activo de regulación hormonal, que libera moléculas metabólicamente activas que pueden inhibir la capacidad del organismo de responder ante la insulina, lo cual se denomina resistencia a la insulina (Graves, D., Liu, R., Oates, T., 2008).

Según Roglic (citado en Robles 2015). La Diabetes Mellitus tipo 2 (DM2) es un creciente problema de salud pública en todo el mundo. Desde 2008, la diabetes se ha clasificado como la séptima causa de muerte en los Estados Unidos, con costos directos anuales estimadas de \$ 245,000,000,000. La Federación Internacional de Diabetes (Roglic, Unwin, 2010) señala que alrededor de 4.6 millones de personas entre 20 y 79 años murieron por causa de diabetes el año 2011, lo que representa $8.2 \%$ de la mortalidad mundial. Lo que respecto a los cálculos para el año 2010 representa un aumento del 13.3\%; antecedentes que aumentan la importancia de una pesquisa temprana, diagnóstico y derivación oportuna. En América Latina, existen 15 millones de personas con Diabetes Mellitus y se espera que esta cifra alcance los 20 millones en 10 años.

Hoy en día, aproximadamente 20.4 millones de adultos estadounidenses tienen diabetes, con un tercio de los casos no diagnosticados. En un estudio realizado en el 2013 por DPTT (Design Features of the Diabetes and Periodontal Therapy Trial), el objetivo fue determinar el efecto de la terapia periodontal no quirúrgico sobre la Hemoglobina Glicosilada (HbA1c) en individuos con DM2 y la periodontitis crónica en comparación con ningún tratamiento. La hipótesis de que los participantes que recibieron la terapia experimentarían un $0.6 \%$ más de reducción de la HbA1c en comparación con los controles no tratados. El DPTT fue un estudio multicéntrico, ensayo de un solo ciego, aleatorizado y controlado de fase III clínica. Los participantes con diabetes moderada-controlada y moderada a la periodontitis avanzadas fueron asignados al azar para recibir tratamiento periodontal inmediata o retardada. Los participantes fueron evaluados al inicio del estudio y a los 3 y 6 meses después de la aleatorización para múltiples medidas de 
control de la diabetes y la periodontitis. El resultado del estudio primario fue el cambio en la HbA1c desde el inicio hasta los 6 meses de la visita después de la aleatorización. Este resultado, demostró controlar el estado de la diabetes y a su vez se asocia la enfermedad periodontal con el riesgo de complicaciones relacionadas con DM. Los resultados secundarios incluyeron: cambio HbA1c desde el inicio hasta la visita de 3 meses, cambio en las medidas clínicas de la periodontitis crónica (índice gingival, sangrado al sondaje, profundidad de sondaje, pérdida de inserción clínica) a los 3 y 6 meses o la profundidad de sondaje, pérdida de inserción clínica y sangrado en sondaje. Son mediciones clínicas estándar utilizadas para evaluar el estado periodontal del individuo (Nunn ME 2004). (Diaz,2010; Smith 2012) Los mecanismos exactos por los que la enfermedad periodontal y su tratamiento pueden afectar el control glucémico en pacientes con diabetes tipo 2 no han sido completamente aclarada. Una hipótesis popular implica vías inflamatorias comunes a la patogénesis de la periodontitis y la resistencia a la insulina. Específicamente, la Interleucina-6 (IL-6) y Factor de Necrosis Tumoral-alfa (TNF-alpha), que están presentes en los tejidos periodontales inflamados, se sabe que afectan adversamente a la señalización de insulina y la acción. Tanto IL-6 y TNF-alpha, pueden entrar en la circulación sistémica en pacientes con periodontitis, donde con el tiempo se pueden alterar la sensibilidad a la insulina en los órganos diana. Por lo tanto, es biológicamente plausible que una periodontitis puede exacerbar la glucemia en los pacientes con DM2 y que el tratamiento, mediante la reducción de los niveles de citoquinas circulantes, pueden mejorar el control glucémico (Goldberg, 2007; Martins, 2009).

\section{Diabetes Mellitus e hiperlipidemia}

La hiperlipidemia es un grupo de trastornos caracterizados por un exceso de lípidos en el torrente sanguíneo. Los pacientes con hiperlipidemia a menudo se manifiestan elevaciones de las Lipoproteínas de Baja Densidad (LDL), Triglicéridos (TRG) y omega-6 ácidos grasos libres. La elevación de ácidos grasos omega-6 ácidos poliinsaturados, a su vez contribuye a la formación de LDL / TRG. La hiperglucemia suele ir acompañada de la hiperlipidemia en

diabetes tipo 1 y tipo 2. Un número de estudios han reportado aumento de colesterol total, TRG, LDL y disminución del HDL en pacientes diabéticos (Xiaodong, Z, et al. 2015). 


\section{Diabetes e inflamación}

(Graves et. al 2008) Tanto la diabetes tipo 1 como la de tipo 2 se asocian con valores elevados de marcadores sistémicos de inflamación. Se ha demostrado en la diabetes un aumento en la concentración sérica del Factor de Necrosis Tumoral (TNF-alpha) y de la Interleucina 6 (IL-6). La DM altera la respuesta inflamatoria frente a los agentes patógenos orales. Por ejemplo, el líquido crevicular gingival humano de pacientes con diabetes tipo $1 \mathrm{y}$ enfermedad periodontal presenta una concentración elevada de Prostaglandina $\mathrm{E}_{2}\left(\mathrm{PGE}_{2}\right)$ y de IL1-BETA, en comparación con el líquido crevicular gingival de pacientes no diabéticos con un grado similar de enfermedad periodontal. Además, los monocitos aislados de pacientes periodontales con diabetes tipo 1 producen cantidades significativamente superiores de TNF-alpha, IL1BETA $\square \square$ y PGE $_{2}$ en respuesta al Lipopolisacárido (LPS), en comparación con pacientes no

diabéticos. ${ }^{14}$ Las funciones biológicas de las citoquinas están relacionadas con la periodontitis, la diabetes y el metabolismo de los lípidos. Varios autores concuerdan que: 1) Las citoquinas están asociadas con resistencia a la insulina y el riesgo de diabetes. 2) Altos niveles de citoquinas pro-inflamatorias en los tejidos periodontales están asociados con el riesgo de periodontitis a causa de sus efectos destructivos de tejidos. 3) Las citoquinas ejercen efectos sobre el metabolismo lipídico al afectar la producción de otras citoquinas, lo que resulta en niveles elevados de ácido graso libre, LDL y TRG. Los efectos de elevación de lípidos en suero se deben al aumento de la producción TRG hepática y/o disminución del aclaramiento TRG.

\section{Diabetes: cicatrización de heridas}

(Torres, 2007; Graves, 2008) Se ha documentado de forma adecuada que la cicatrización de heridas sufre un retraso o es incompleta en las personas diabéticas, lo que también se ha demostrado en modelos animales con diabetes. Se han propuesto varios mecanismos celulares, como la reducción o la disfunción de Leucocitos Polimorfonucleares (PMN) y macrófagos, el mantenimiento de la expresión de citocinas y del infiltrado de células inflamatorias, la reducción en la producción de factores de crecimiento, la disminución de la proliferación celular y de la síntesis de la matriz extracelular, así como el aumento en la producción de enzimas proteolíticas (Figura 1). 
Figura 1. Mecanismo a través de los que la diabetes puede influir en la progresión de la enfermedad periodontal

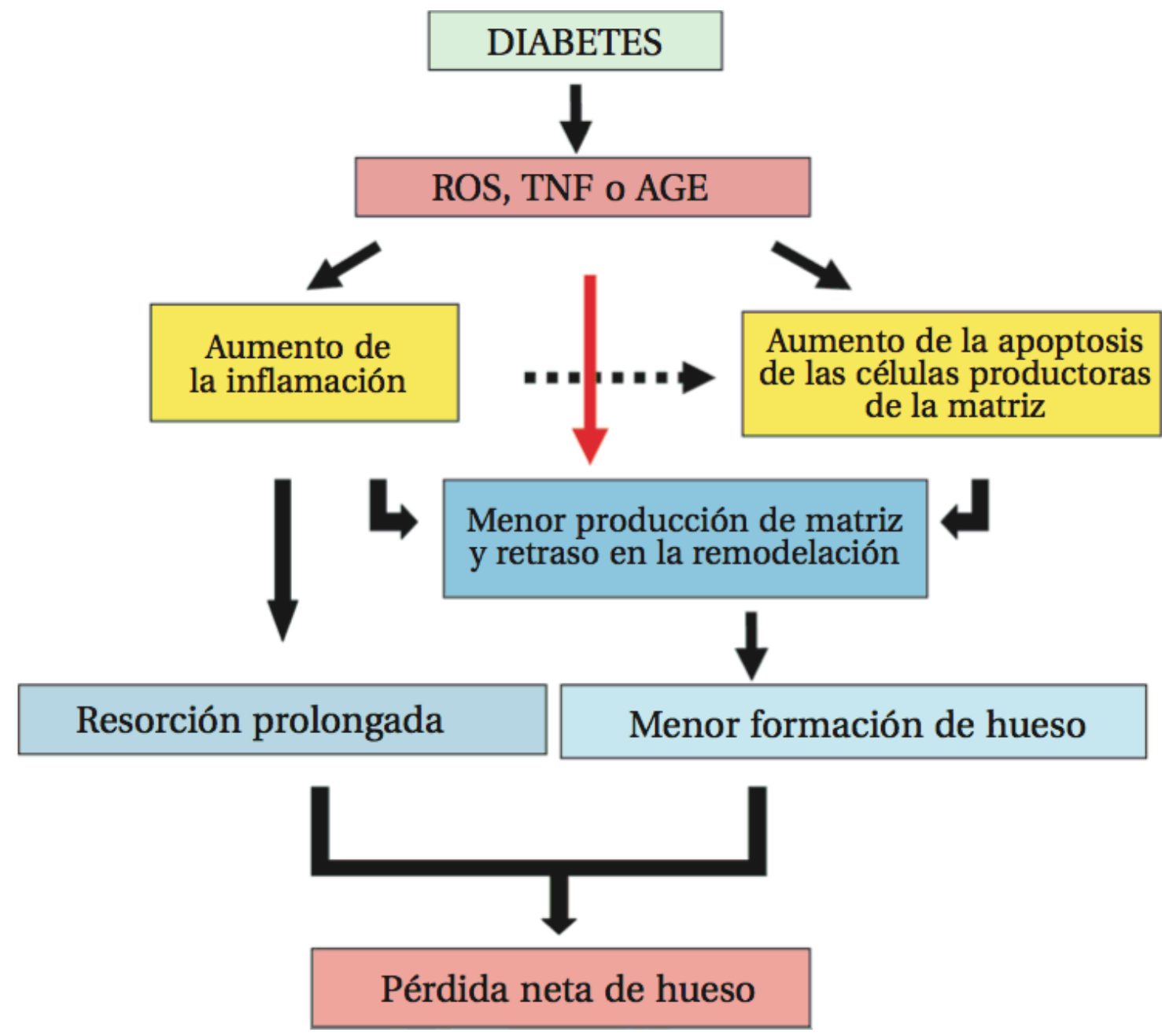

Fuente: (Graves et. al 2008).

La síntesis, la maduración y la homeostasis del colágeno parecen estar afectadas por los niveles de glucosa. Además de encontrar disminución de la producción de colágeno en asociación con la diabetes, los investigadores también han encontrado una mayor actividad de colágenasa en el tejido gingival en los animales (Kumar M., Mishra L., Mohanty R., Nayak R., 2014). En algunos estudios se demostró que la glicosilación del colágeno preexistente en los márgenes de la herida disminuye su solubilidad alterando su remodelación y además por otro lado la mayor actividad colagenasa existente en el diabético degrada el nuevo colágeno sintetizado retrasando y dificultando todo ello la cicatrización de la herida. (Torres et al 2007). 
Mealey \& Ocampo (2008) afirmaron que el impacto potencial de la elevación de mediadores pro-inflamatorios sistémicos en pacientes con diabetes es alto. La inflamación sistémica es significativamente elevada en presencia de obesidad, resistencia a la insulina, hiperglucemia, y diabetes.

(Graves et al. 2008) Se llevo acabo una investigación por He H. y Liu R. en el 2004, en ratones diabéticos y normales para conocer la respuesta de cicatrización tras la destrucción tisular inducida por Porphyromonas Gingivalis. Se provoco una reacción inflamatoria ya que se inoculó Porphyromonas Gingivalis muerto en el cuello cabelludo de los ratones, los resultaron arrojaron que la Diabetes Mellitus modifica la respuesta frente a las bacterias aumentando el número de fibroblastos positivos para la caspasa 3 activada, así como el numero de apoptosis de los fibroblastos a la hora de la cicatrización máxima. Por tanto, el incremento de apoptosis de estas células coincidía con la reducción en el numero de fibroblastos y con la disminución en la capacidad productora de matriz, por tanto mayor retraso en la remodelación y reestructuración, causando resorción prolongada, menor formación de hueso, por tanto obteniendo perdida neta de hueso.

(Al-Mashat, H., Kandru, S., Liu, R., Behl, Y., Desta, T. \& Graves DT. 2006) El incremento de la apoptosis de estas células coincidía con la reducción en el número de fibroblastos y con una disminución en la capacidad productora de matriz. La diabetes también originaba una inducción global de genes proapoptóticos durante el proceso de reparación. De un total de 276 genes apoptóticos examinados, 71 genes aumentaban el doble o más, de modo que 63 de ellos eran proapoptóticos y 8 antiapoptóticos. Esto señala a otro mecanismo por el que la diabetes puede interferir con la capacidad de reparar el daño tisular, al potenciar la muerte de células productoras de la matriz.

\section{Cómo identificar un paciente diabético}

Los dentistas a menudo no son conscientes de los detalles de la situación y la gestión de DM en su paciente, y los médicos no suelen evaluar la condición periodontal de sus pacientes. Por eso es importante seguir estos pasos que ayudaran a dar un diagnóstico temprano: 
- Es importante que el operador realice una anamnesis detallada y profunda.

- Ser directo con el paciente en las preguntas que involucren a DM si se sospecha que la padece. ¿Qué tipo de diabetes padece? ¿Desde cuándo la padece? ¿Esta tomando medicamento? ¿Fecha ultima de estudio clínico? ¿Valores de HbA1c que maneja? ¿Cuántos más en su familia la presentan?

- Observar al paciente desde el momento que ingresa a la consulta si viene tembloroso, agitado, con ansiedad, con transpiración o taquicardia.

- Observarle las extremidades por si presenta descamación o piel seca o amoratada.

- Pesar y medir al paciente, y evaluar su estado físico.

- Realizar una revisión exhaustiva bucal. Se puede observar una serie de cambios en boca en la influencia de la DM sobre el periodonto tales como: agrandamiento gingival, pólipos gingivales sésiles o pediculados, proliferaciones gingivales polipoides, formación de abscesos, periodontitis y perdida de soporte óseo dental, mayor perdida de inserción, movilidad dentaria, hemorragia espontanea al sondeo. Dentro de las alteraciones secundarias se puede observar alteraciones bucales como: un mayor índice de caries, queilosis, desecamiento y agrietamiento de las mucosas, ardor bucal y lingual, menor flujo salival y alteraciones de la flora bucal, predominio de Candida Albicans, estreptococos hemolíticos y estafilococos.

Realizar pruebas de laboratorio para diagnostico:

Tabla 1. Prueba de hemoglobina glicosilada (HbAlc).

\begin{tabular}{cc}
\hline Porcentaje & Estado \\
\hline $4-6 \%$ & Normal \\
$<7 \%$ & Control adecuado de DM \\
$7-8 \%$ & Control moderado de DM \\
$>8 \%$ & Mejorar el control de DM
\end{tabular}

Criterios de la American Diabetes Association para el diagnóstico de la Diabetes Mellitus, alteración de la tolerancia a la glucosa (ATG) y alteración de la glucosa en ayunas (AGA). 
1. Síntomas de diabetes más glucosa plasmática aleatoria (sin ayuno) $\geq 200 \mathrm{mg} / \mathrm{dl}$. La glucosa aleatoria puede obtenerse en cualquier momento del día desde la última comida.

2. Glucosa plasmática en ayuno $\geq 126 \mathrm{mg} / \mathrm{dl}$. Lo normal en ayuno es $70-100 \mathrm{mg} / \mathrm{dl}$.

3. Glucosa postprandial de dos horas $\geq 200 \mathrm{mg} / \mathrm{dl}$ durante una prueba de tolerancia de glucosa oral. Lo normal es $<140 \mathrm{mg} / \mathrm{dl}$.

Manifestaciones bucales y periodontales de la enfermedad

Diabéticos incontrolados:

1. Sequedad de la boca.

2. Eritema difuso de la mucosa.

3. Lengua saburral y roja, con indentaciones marginales.

4. Tendencia a formación de abscesos periodontales.

5. "periodontoclasia diabética" y "estomatitis diabética".

6. Encía agrandada.

7. Pólipos gingivales sésiles o pediculados.

8. Papilas gingivales sensibles, hinchadas, que sangran profundamente.

9. Proliferaciones gingivales polipoides y aflojamiento de dientes.

10. Mayor frecuencia de la enfermedad periodontal con destrucción alveolar tanto vertical como horizontal.

11. Los diabéticos poseen una menor resistencia a las infecciones, aunque no está claro si poseen una frecuencia real mas elevada de infecciones, o si una vez contraídas, las infecciones prosperan. Esta susceptibilidad a las infecciones resultaría ser una combinación de microangiopatía, acidosis metabólica y fagocitosis ineficaz de los macrófagos. 
12. Alteraciones en la flora de la cavidad oral con mayor predominio de Candida Albicans, Estreptococo Hemolítico y Estafilococo.

13. Patrones de erupción alterados.

14. Aumento de sensibilidad dentaria a la percusión.

15. Aumento de la incidencia de hipoplasia del esmalte.

16. Aumento de la incidencia de caries (Torres 2007; Artuña).

\section{Tratamiento periodontal en diabéticos}

Aunque algunos estudios no lograron observar la relación entre el grado de control de la glucemia y la periodontitis, la mayoría de los estudios generalmente apoyan que el buen control de la glucemia disminuye la severidad de la periodontitis, mientras que un mal control de la glucemia aumenta el riesgo de periodontitis (Bustamante 2013).

\section{¿Responden bien los pacientes diabéticos al tratamiento periodontal?}

Numerosos estudios de sujetos con DM2 y periodontitis han mostrando mejoría en el control metabólico (disminución en 0.9 -1\% de la Hemoglobina glicosilada [HbA1c]) luego de ser sometidos a tratamiento periodontal convencional, adicionando o no antibióticos, probablemente debido a la disminución de IL-6, TNF-alpha, reacción en cadena de la polimerasa (PCR), leptina y el incremento de los niveles de adiponectina (Bustamante 2013). Estudios recientes, muestran que el tratamiento periodontal no quirúrgico intensivo (6 horas de detartraje y pulido radicular con un instrumento ultrasónico) produce aumento significativo de la respuesta inflamatoria sistémica en sujetos con periodontitis y buena salud general (Pavez et. al 2011).

- Como parte de su evaluación inicial, los pacientes con diabetes tipo 1, tipo 2 y gestacional (GDM) deben recibir un examen oral completo que incluya un minucioso examen periodontal (Zerón, A 2013).

- Se le debe solicitar al paciente una prueba de Hemoglobina Glicosilada (HbA1c). 
- El paciente debe llevar su medidor de glucosa en cada visita.

- Traer siempre su medicamento a la mano.

- Si se realizara un procedimiento quirúrgico, se necesitara aprobación del medico tratante.

- Pedirle al paciente que siempre que asista a consulta, vaya con ingesta de alimentos.

- Realizar profilaxis para disminuir la carga bacteriana.

- Técnicas de cepillado y cuidados orales.

- Uso de clorhexidina al $0.12 \%$

- Trabajar por sextantes para reducir el tiempo de trabajo.

- Realizar Fase I no quirúrgica, raspado y alisado, eliminando carga bacteriana y focos infecciosos (extracción restos radiculares) (Pavez et. al 2011).

- Azitromicina 500 mg cada 24 horas por tres días (Pavez et. al 2011). Las tetraciclinas en dosis bajas y las tetraciclinas modificadas químicamente han demostrado disminuir significativamente la producción de colagensasa y degradación del colágeno. Tales como: doxicilina, la minociclina y el clorhidrato de tetraciclina estas tienen posibles beneficios en la inhibición del inicio y progresión de la periodontitis, aunque no se ha publicado sobre su uso en pacientes diabéticos.

- Revaloración de la Hemoglobina Glicosilada después del tratamiento.

- Revaloración para una cirugía de desbridamiento por colgajo si el tratamiento así lo requiera.

- Se recomienda hacer una revisión periodontal anualmente. 


\section{Justificación}

Existe una gama de estudios documentados, en donde enfermedades sistémicas como la Diabetes Mellitus, tienen una relación estrecha con la salud bucal, modificando la respuesta del huésped a los microorganismos periodontales y exacerbando la enfermedad periodontal. Es por ello que creemos justificado realizar este estudio en base a los conocimientos científicos obtenidos de la selección de datos recopilados, con el fin de conocer, comprender y analizar los problemas sistémicos y bucales que relacionan a la enfermedad periodontal con la Diabetes Mellitus.

\section{Objetivos}

- Identificar la relación bidireccional ente la enfermedad periodontal y la Diabetes Mellitus.

- Conocer los dos tipos de enfermedades de una manera individualizadas, para posteriormente identificar con exactitud como exacerba una enfermedad a la otra y viceversa.

- Identificar si la Diabetes Mellitus es un factor de riesgo para presentar enfermedad periodontal.

- Identificar si la enfermedad periodontal es un factor de riesgo para presentar Diabetes Mellitus.

- Conocer los estadios y problemas presentes en la cicatrización de la herida, en un paciente con diabetes y periodontitis.

- Identificar las diferentes manifestaciones bucales y sistémicas de un paciente con problemas periodontales y con Diabetes Mellitus.

- Dar a conocer más a opciones de los cuidados y lineamientos que se deben tener al tratar a estos pacientes para brindarles una atención medica-dental con suficiente apoyo científico y mejores resultados clínicos. 


\section{Metodología}

Para la realización de esta revisión bibliográfica, se realizo una búsqueda profunda en base de datos (pubmed, periodontology 2000, journal of periodontology, medline, scielo, elsevier, intramed, j periodontol, google académico) nacionales e internacionales. Se obtuvieron datos de la biblioteca de la Facultad de Odontología Mexicali, tanto material físico como digital. Utilizando palabras claves como: Diabetes Mellitus, periodontitis, hiperlipidemia.

Se obtuvieron 58 artículos, de los cuales se descartaron 18, con base en los criterios de inclusión y exclusión: 25 son revisión bibliográfica, 3 son resultado de experimentos aplicados al grupo control, 1 meta-análisis, 1 estudio de caso-control clínico, 1 estudio de sección transversal, 1 estudio observacional descriptivo, 1 ensayo clínico, multicéntrico, ciego, aleatorizado, controlado, 1 estudio epidemiológico, 2 trabajo de investigación, 2 datos obtenidos de paginas de internet y 2 revisiones de consenso (Sociedad Española de Periodoncia [SEPA], Academia Americana de Periodoncia [AAP], Federación Europea de Periodoncia [EFP]). Donde se excluyeron artículos con idioma diferente al inglés o español, artículos que estaban repetidos en otra base de datos, artículos donde no menciona la relación de ambas enfermedades, artículos mayores a diez años.

Entre los criterios de inclusión se tomó la referencia de artículos no mayores de 10 años (20062016), acceso gratuito y paga, idioma inglés y español, estudios en humanos y animales, que el texto hablara de la relación diabetes-periodontitis.

\section{Resultados}

Los resultados nos arrojan que sí existe una relación bidireccional entre Periodontitis y Diabetes Mellitus, en la cual una enfermedad ayuda a exacerbar a la otra, la mayoría de los autores concuerdan en la información recopilada, aunque hacen falta más investigaciones sobre como el tratamiento de la enfermedad periodontal ayuda positivamente a nivelar el control metabólico de la diabetes. 
La Diabetes Mellitus es considerada un trastorno metabólico que debe ser detectado según los criterios diagnósticos propuestos por la Asociación Americana de Diabetes; también es importante que los pacientes que padecen la enfermedad tengan un control adecuado de su glucemia.

(Ghiotto G. Et al 2012) Los estudios hasta el momento han demostrado una relación bidireccional entre la Diabetes Mellitus y la enfermedad periodontal, por ello el profesional médico y odontológico deben conocer estas relaciones para el adecuado diagnóstico y tratamiento de los pacientes.

(Martins N. et al 2007) Este meta-análisis de 57 estudios revisados por pares conduce a la conclusión de que la DM de tipo 2 es un factor de riesgo para la periodontitis. La diferencia global para CAL de $1.0 \mathrm{~mm}$ (IC 95\%: 0.15 a 1.84, P < 0.021) fue significativa. Además, los estudios longitudinales revelaron significativamente más progresión de la enfermedad periodontal entre los diabéticos tipo 2 en comparación con los no diabéticos. Sin embargo, la evidencia de una asociación entre el DM tipo 1 y la periodontitis es insuficiente.

(Kumar M. et al 2014) Las enfermedades periodontales y la Diabetes Mellitus están estrechamente asociadas y son enfermedades crónicas altamente prevalentes con muchas similitudes en la patobiología. La diabetes aumenta claramente el riesgo de enfermedades periodontales, y los mecanismos biológicamente plausibles se han demostrado en abundancia. Menos claro es el impacto de las enfermedades periodontales sobre el control glucémico de la diabetes y los mecanismos a través de los cuales esto ocurre. Se necesitan más investigaciones para aclarar este aspecto de la relación entre las enfermedades periodontales y la diabetes.

(Fajardo M. et al 2016) La Diabetes Mellitus constituye un factor de riesgo para el desarrollo de la enfermedad periodontal, pero esta última puede poseer efectos negativos sobre los valores de la glucemia. Las formas más graves de enfermedad periodontal se han asociado a niveles elevados de productos de glucosilación avanzada. Por tanto, el mal control metabólico mantenido en personas diabéticas contribuye al desarrollo y la progresión de la enfermedad periodontal.

(Ross JH. et al 2010) En sus estudios recientes han demostrado que la glucosa aumentó la secreción de TNF-alpha, IL1-BETA e IL-6 estimulada por LPS de macrófagos U937 humanos 
por 6, 4 y 27 veces, respectivamente. Obviamente, el aumento de la secreción de IL-6 estimulada por LPS con glucosa alta es mucho mayor que la de la secreción de TNF-alpha e IL1-BETA, lo que sugiere un papel importante de la IL-6 en la enfermedad periodontal asociada a la diabetes.

Las relaciones descritas en este artículo conecta la dieta, la obesidad, la diabetes y la periodontitis sugiere que todos los profesionales de la salud trabajen juntos para tratar mejor la salud general de la población mundial, requiriendo un enfoque multidisciplinario para la prevención, diagnóstico temprano y atención óptima.

La enfermedad oral más importante asociada con la diabetes es el aumento de la prevalencia y la gravedad de la periodontitis. El control glicémico pobre persistente se ha asociado con la incidencia y la progresión de la gingivitis, la periodontitis, y la pérdida del hueso alveolar.

(Ryan M. et al 2016) Una extensa revisión de la literatura revela que hay evidencia mundial de que la diabetes afecta negativamente a la salud periodontal.

(Bascones-Martines A. et al 2014) Ésta claramente establecido que el control glucémico mejora el estado periodontal y el control de la infección periodontal mejora la glucemia en los pacientes diabéticos, la implicación clínica de este acontecimiento aún debe ser investigada. Sin embargo, la posibilidad de que ambas enfermedades puedan estar compartiendo algunos aspectos patogénicos comunes debe tenerse siempre en mente para garantizar el diagnóstico precoz de ambas.

(Oana A. et al 2013) La inflamación periodontal conduce a un aumento de las citocinas circulantes, mediadores inflamatorios y la respuesta autoinmune a la infección. Se ha demostrado que la gravedad de la destrucción periodontal está relacionada con los efectos de control de la glucemia, estando también implicados otros factores. En los pacientes diabéticos con enfermedad periodontal, la IL1-BETA, la IL-6 y el TNF aumentan gradualmente con la evolución de la Diabetes Mellitus, al igual que lesiones óseas periodontales. También se ha informado de una correlación significativa entre el control metabólico y la gravedad y extensión de las lesiones periodontales. 
(Vásquez E \& López M. 2014) Demuestra en un caso clínico de una paciente con diabetes mal controlada tipo 1 y periodontitis agresiva generalizada, como ayuda el buen control glicémico a mejorar el tratamiento periodontal. Las pruebas de laboratorio finales revelaron un control de la glicemia de $140 \mathrm{mg} / \mathrm{dL}$, comparándolo con las cifras iniciales de $220 \mathrm{mg} / \mathrm{dl}$. El tratamiento periodontal no presentó ninguna complicación y el control metabólico de su diabetes dio origen a un mejoramiento significativo en la condición periodontal.

(Taylor GW \& Borgnakke WS 2008) Los estudios observacionales proporcionaron evidencia consistente de mayor prevalencia, gravedad, extensión o progresión de al menos una manifestación de la enfermedad periodontal en 13/17 informes revisados.

El objetivo de esta revisión no sistemática fue explorar y aclarar las interrelaciones $e$ interacciones entre las enfermedades periodontales y cuatro enfermedades sistémicas comunes, a saber, la diabetes, las enfermedades respiratorias, las enfermedades cardiovasculares y la osteoporosis.

(Lan-Chen Kuo et al. 2008) La exposición prolongada a la hiperglucemia es el principal factor responsable de las complicaciones diabéticas. Las enfermedades periodontales están bien establecidas como una complicación de la diabetes, lo que sugiere que los pacientes con diabetes son más propensos a desarrollar enfermedades periodontales.

(Corbi S et al. 2014) Hasta donde sabemos, este es el primer estudio que investiga la asociación entre el daño del ADN mediante el ensayo CBMN y el examen físico, las mediciones bioquímicas (control metabólico y lipoproteína pro) y los parámetros clínicos periodontales en pacientes que tienen o no, 2 diabetes, dislipidemia y enfermedad de Parkinson. Los resultados presentados aquí indican una asociación entre la diabetes tipo 2 y el daño del ADN, con respecto a la frecuencia de las células binucleadas con micronúcleos (MCF), MNF y puentes nucleoplásmicos.

(Galvis M. et al 2012) Existe una relación bidireccional entre la Diabetes Mellitus y la enfermedad periodontal, ya que los estudios actuales muestran cómo un pobre control glicémico ayuda a exacerbar la enfermedad periodontal, al igual que un buen tratamiento de esta ayuda en el control glicémico de la diabetes. Dicha relación ha tratado de ser explicada a través de diversas teorías, ya sean inmunológicas, micro-biológicas o etiopatológicas. Todo 
apunta a que los protagonistas de dicha relación bidireccional son los AGE, debido a que participan en el proceso inmunológicco de ambas enfermedades.

\section{Conclusiones}

- La enfermedad periodontal es una enfermedad multifactorial y uno de los factores de riesgo mas importantes es la Diabetes Mellitus.

- Es importante que el dentista y otros profesionales de la salud tengan un conocimiento profundo de la diabetes, por su amplia prevalencia, la obesidad ésta claramente asociada a un aumento del riesgo de desarrollar diabetes, a medida que se incrementan la prevalencia y la incidencia de diabetes, también lo hacen los costos, tanto financieros como en morbilidad y mortalidad.

- Los pacientes con diabetes deben saber que el riesgo de enfermedad periodontal aumenta con una diabetes mal controlada. También deben saber que si sufren de enfermedad periodontal, el control de su glucemia puede ser más difícil de manejar y estarán en mayor riesgo de complicaciones de la diabetes.

- El medico y odontólogo deben estar siempre relacionados y trabajar en equipo para brindarle los cuidados necesarios al paciente con DM y evitar así un choque hipoglucémico o una diseminación infecciosa grave. 


\section{Bibliografía}

Al-Khabbaz, A., Al-Shammari, K., Hasan, A., Abdul-Rasoul, M., Periodontal health of children with type 1 diabetes mellitus in Kuwait: a case-control study. Med Princ Pract 2013;22(2):144-9.

Al-Mashat, H., Kandru, S., Liu, R., Behl, Y., Desta, T. \& Graves DT. Diabetes enhances mRNA levels of proapoptotic genes and caspase activity, which contribute to impaired healing. Diabetes 2006: 55: 487-495.

Artuña, V., Manifestaciones orales de la diabetes. URL, http://www.clinidabet.com/es/info/diabetes/02educacion/07educando/01.htm.

Bascones-Martínez A., Muñoz-Corcuera M., Bascones-Ilundain J. Diabetes y periodontitis: una $\begin{array}{lllll}\text { relación } & \text { bidireccional, } & \text { Med } & \text { Clin } & \text { (Barc). }\end{array}$ http://dx.doi.org/10.1016/j.medcli.2014.07.019

Bustamante, G., Diabetes y enfermedad periodontal, Rev, act. Clin. Mod.v.31 la paz mayo 2013.

Corbi S., Bastos A., Orrico S., Secolin R., Dos Santos R., Takahashi C., Scarel-caminaga R., Elevated micronucleus frequency in patients with type 2 diabetes, dyslipidemia and periodontitis, mutagenesis advances Access published september 18,2014, pp. 1-7, doi: 10.1093/mutage/geu043

Díaz, R., Villegas, F., Diabetes e infección periodontal. Intramed, Revista nacional de odontología año 2 vol. VII, 2010.

Domínguez, V., Calatrava, L., Ortega, P., Astasio, P., Pérez, L., de pereda, A. \& García, J. Enfermedad periodontal en diabéticos juveniles y no diabéticos, Rev. San hig púb 1993, vol 67 no.6

Fajardo M, Rodríguez O, Hernández M, Mora N. Diabetes mellitus y enfermedad periodontal: aspectos fisiopatológicos actuales de su relación. Rev. Scielo, Medisan Vol.20 No.6 Santiago de Cuba jun 2016. 
Fernandes, J., Wiegand, R., Salinas, C., Grossi, S., Sanders, J., Lopes-Virella, M., et al. Periodontal disease status in Gullah African americans with type 2 diabetes living in south Carolina. J Periodontol 2009;80:1062-8.

Galvis M., Montoya Y., Saldarriaga A., diabetes y enfermedad periodontal: hacia un modelo clínico bidireccional, revista nacional de odontología, volumen 8, No 14, enero-julio 2012.

Ghiotto G, López R, Tineo M, Villareal L, Alarcón M, Diabetes mellitus y enfermedad periodontal. Revisión bibliográfica de la situación actual. Rev. estomatol Heradiana. 2012, 22 (3)183-8.

Graves, D., Liu, R., Oates, T., Inflamación y apoptosis potenciadas por la diabetes: impacto sobre la salud periodontal, Periodontology 2000 (Ed Esp), Vol. 20, 2008, 80-86.

Goldberg, M., Han, Y., Yan, C., Shaw, M., Garner, W., TNF alpha supresses alpha-smooth muscle actin expression un human dermal fibroblastos, an implication for anormal wound healing. J invest dermatol, 2007,127: 2645-2655.

Herrera D., Rodríguez-Casanovas H., Herrera J., Informe SEPA/ FUNDACIÓN SED, diabetes y enfermedades periodontales.

Jiménez, T., Licea, M., Relación entre diabetes mellitus y enfermedad periodontal, revista Peruana de epidemiologia vol 17, núm. 3, diciembre 2013, pp 1-7. Recuperado de http://www.redalyc.org/articulo.oa?id=203129459002

Kumar M., Mishra L., Mohanty R., Nayak R., Diabetesand gum disease: the diabolic duo, Diabetes \& metabolic síndrome: Clinical research \& reviews. elsevier 8 (2014) 255258. Doi: http://dx.doi.org/10.1016/j.dsx.2014.09.022

Kuo L., Polson A., Kang T., Associations between periodontal diseases and systemic diseases: a review of the inter-relationships and interactions with diabetes, respiratory diseases, cardiovascular diseases and osteoporosis, elsevier public health 2008, 122, 417-433 doi:10.1016/j.puhe.2007.07.004 
Martins, N., Vianna, M., Sansone, C., Sheiham, A., The relationship between diabetes mellitus and destructive periodontal disease: Meta-analysis, Oral health prev dent 2009; 7:107127.

Mealy, B., Ocampo, G., Diabetes mellitus y enfermedad periodontal. Periodontology 2000. 2008: 18(1);86-104.

Miguel, P., Niño, A., Batista, K., Diabetes mellitus y enfermedad periodontal. Rev. Scielo, ccm vol.20 no. 2 holguín abr-jun 2016.

Nunn ME, interpretación de la etiología de la periodontitis: resumen de los factores de riesgo periodontales, periodontology 2000 (Ed Esp), Vol. 7, 2004, 11-23.

Organización Mundial de la Salud OMS (1999). http://www.who.int/diabetes/actiononline/basics/es/

Pavez, V., Araya, A., Baksai, N., Respuesta al tratamiento periodontal de diabéticos tipo 2 con mal control metabólico y obesos intolerantes a la glucosa, con periodontitis severa, Rev. Clin. Periodoncia Implantol. Rehabil. Oral Vol. 4(2); 50-53, 2011.

Piyamas, A., Piyawan, A., Suwimol, T., Quantification of key periodontal pathogens in insulindependent type 2 diabetic and non-diabetic patients with generalized chronic periodontitis, Anaerobe 22 (2013) 64e68. Recuperado de http//www.elsevier.com/locate/anaerobe

Robles, S., Rosas, C., Herrera, L., Rivas, N., Diabetes y periodontitis, conocimiento en profundidad de la salud de Valdivia 2013. Revista dental de Chile 2015, 106(1)4-8.

Roglic, G., Unwin, N., Mortality attributable to diabetes: estimates for the year 2010. Diabetes Res Clin Pract. 2010 Jan;87(1):15-9.

Ross J.H., Hardy D.C., Schuyler C.A., Slate E.H. Mize T.W., Huang Y. Expression of periodontal interleukin-6 protein is increased across patients with neither periodontal disease nor diabetes, patients with periodontal disease alone and patients with both diseases. J periodont Res 2010; 45:688-694. 
Ryan M., Raja V., Diet, obesity, diabetes, and periodontitis: a syndemic approach to management. Curr Oral Health Rep (2016) 3:14-27, Doi: 10.1007/s40496-016-0075-1

Smith, P., Retamal, I., Cáceres, M., Romero, A., Silva, D., Arancibia, R. \& Martínez, C., Diabetes y su impacto en el territorio periodontal. Rev clin, periodoncia Implanton. Rehabil oral Vol.5 (2) 90-92,2012.

Tan, W., Tay, F., Lim, L., Diabetes as a risk factor for periodontal disease: current status and future considerations. Ann Acad Med Singap 2006;35; 571-81

Taylor GW., Borgnakke WS., Periodontal disease: associations with diabetes, glycemic control and complications, oral diseases 2008, 14, 191-203. Doi: 10.1111/j.16010825.2008.01442.x

The DPTT study group, S. Engebretson, M. Gelato, L. Hyman, B.S. Michalowicz, E. Schoenfeld, Design features of the Diabetes and Periodontal Therapy Trial (DPTT): A multicenter randomized single-masked clinical trial testing the effect of nonsurgical periodontal therapy on glycosylated hemoglobin (HbA1c) levels in subjects with type 2 diabetes and chronic periodontitis, Contemporary Clinical Trials 36 (2013) 515-526. Recuperado de http//www.elsevier.com/locate/conclintrial.

Torres, M., Díaz, M., la diabetes mellitus y su vinculación en la etiología y patología de la enfermedad periodontal. Gaceta medica espirituana 2007, 9 (2).

Vásquez E., López M., Tratamiento multidiciplinario en una paciente con periodontitis agresiva generalizada y diabetes mellitus tipo 1, revista odontológica mexicana, vol.18, núm. 1, enero-marzo 2014, pp32-37

Velea O., Kralev C., Onisei D., Onisei D., Nica L., Velea I., European Scientific Journal march 2013 edition vol.9, N0.9 ISSN: 1857-7881

Weinspach, K., Staufenbiel, I., Memenga-Nicksch, S., Ernst, S., Geurtsen, W., Gunay, H. Level of information about the relationship between diabetes mellitus and periodontitis results from a nationwide diabetes information program. Eur J Med Res 2013;18:6. http://dx.doi.org/10.1186/2047-783x-18-6. 
World Health Organization. Oral Health Fact Sheet. n. 318, April 2012. Public health solutions for oral diseases are most effective when they are integrated with those for other chronic diseases and with national public health programmes. The WHO Global Oral Health Programme aligns its work with the strategy of chronic disease prevention and health promotion. Emphasis is put on developing global policies in oral health promotion and oral disease prevention.

Xiaodong, Z., Wenyi, Z., Xiaoli, L., Wu, Z., Yiming, L., Interrelationship between diabetes and periodontitis: Role of hyperlipidemia, archives of oral biology 60 (2015) 667-674. Recuperado de http://www.elsevier.com/locate/aob

Zerón, A., Consenso - Enfermedades Periodontales y Enfermedades Sistémicas, medigrafic, revista Mexicana de periodontología, Vol. III, 2013, Núm. 3. Pp. 99-108. Recuperado de www.medigraphic.com/periodontologia 